\title{
Obesity and Pancreatic Cysts in African American Patients
}

Anahita Shahnazi ${ }^{1}$, Dilhana Badurdeen ${ }^{2}$, Adeyinka O. Laiyemo ${ }^{2}$, Mehdi Nouraie ${ }^{3}$, Hassan Brim ${ }^{4}$, Priscilla Wessly ${ }^{1}$, Sahar Geramfard ${ }^{1}$, Ali Afsari ${ }^{5}$, Niel Page ${ }^{1}$, Hassan Ashktorab ${ }^{2}$

1. Department of Medicine, Howard University Hospital, Washington DC, USA 2. Department of Medicine \& Cancer Center, Howard University College of Medicine, Washington DC, USA 3. Department of Medicine, University of Pittsburgh Medical Center, Pittsburgh, USA 4. Department of Pathology, Howard University College of Medicine, Washington DC, USA 5. Pathology, Howard University, Washington DC, USA

Corresponding author: Anahita Shahnazi,dr.shahnazi@gmail.com

\section{Abstract}

\section{Objective}

Obesity is one of the risk factors for pancreatic cancer and a prognostic factor for acute-chronic pancreatitis.

\section{Aim}

To explore the relationship and association between obesity and pancreatic cysts over a 25 -year period in African American patients.

\section{Methods}

We reviewed the medical records of 207 patients diagnosed with pancreatic cysts via radiology and pathology data from January 1988 to December 2012. A control group was selected from a separate group of healthy patients without a history of pancreatic disease. The patients were evaluated in five groups according to the last 20 years of diagnosis in five-year intervals.

\section{Results}

Most patients with pancreatic cyst ( $73 \%$ ) were overweight (defined as a body mass index (BMI) $\geqslant 25$ ), and $53 \%$ had a history of chronic pancreatitis compared to patients in the control group. There was a significant difference between the two groups; $79 \%$ of patients group were overweight (BMI $\geqslant 25$ ) vs. $66 \%$ in control group $(p=0.02)$. The incidence of obese and overweight patients was significant $(85 \%)$ during the 2008 to 2012 interval for the test group $(\mathrm{p}=0.009)$.

\section{Conclusion}

Received 06/29/2018 Review began 07/27/2018 Review ended 08/18/2018 Published 08/20/2018

\section{() Copyright 2018}

Shahnazi et al. This is an open access article distributed under the terms of the Creative Commons Attribution License CC-BY 3.0., which permits unrestricted use, distribution, and reproduction in any medium, provided the original author and source are credited.
Given the increasing proportion of obese pancreatic cyst patients in recent decades compared to the proportion noted in the 1990 s, obesity plays a large role in the formation of pancreatic cysts.

Categories: Internal Medicine, Gastroenterology

Keywords: pancreatic cyst, pseudocyst, obesity

\section{Introduction}

Obesity is one of the risk factors of pancreatic cancer and is a prognostic factor for acute and chronic pancreatitis $[1,2]$. Pancreatic pseudocysts of the pancreas result from pancreatic inflammation and necrosis. They comprise $15 \%$ to $30 \%$ of pancreatic cysts overall $[1,3]$ and close to $50 \%$ of pancreatic cysts in patients with a history of chronic pancreatitis [4]. There are some risk factors associated with pancreatic cysts and obesity, and their mechanisms have been studied [5]. Recently, visceral fat and adipocytokines were shown to play very important roles in a variety of conditions, including inflammation [5-9]. Obesity can cause inflammation and fatty necrosis that can lead to pancreatic pseudocyst production [6].

The role of obesity and its association with the development of pancreatic cysts has not been well evaluated, especially in African American (AA) patients, and current data are conflicting. Therefore, we aimed to determine the frequency of obesity in African American patients with pancreatic cysts.

\section{Materials And Methods}

In this retrospective study, we reviewed the medical records of 207 referred African American patients with chronic abdominal pain, nausea and vomiting, early satiety, and weight loss during the period from January 1988 to December 2012 who were also diagnosed with pancreatic pseudocysts by some attending gastroenterologist at Howard University Hospital based on radiological, clinical, laboratory, and pathology 


\section{Cureus}

data. In this study, pancreatic pseudocysts were mainly detected by abdominal computed tomography (CT) scans. Cyst had characteristic features such as a well-defined wall, homogeneous fluid density, and no nonliquid components. CT scan with intravenous contrast medium and pathology reports helped to diagnose pancreatic cystic neoplasms. The study was approved by Howard University Institutional Review Board.

We used frequency and median (interquartile range) to show the distribution of categorical variables and age. We used the Kruskal-Wallis or Chi-square test to determine the association between clinical variables and pancreatic cystic neoplasms or chronic pancreatitis. Statistical analysis was done by STATA 13.0 (StataCorp., College Station, TX).

\section{Results}

Pancreatic cyst in African American patients comprised the case group, and a control group was selected from healthy African American patients without a history of pancreatic disease and had normal results on a colonoscopy screening.

The median ages for the case group and control group were 51 years (range, 45 to 59 years) and 57 years (range, 51 to 63 years). Sixty-two percent of patients in the case group were men, and $46 \%$ of patients in the control group were men. In the case group, $0.9 \%$ of the patient group had pancreatic cystic neoplasms, and the others had pseudocysts. There was a significant difference between the two groups; $79 \%$ of the case group were overweight (Body mass index $(\mathrm{BMI}) \geqslant 25)$ vs. $66 \%$ in the control group $(\mathrm{p}=0.02)$. Also, $20 \%$ of patients in the case group were obese with a BMI $>30$.

Most of the patients $(55 \%, 114 / 207)$ with pancreatic pseudocyst had a history of pancreatitis. The control group had significantly more patients who smoke (79\% in the control group vs. $66 \%$ in the case group (p= 0.02)), but alcohol consumption was not significantly different between the two groups (Table 1).

\begin{tabular}{|c|c|c|c|}
\hline & Healthy controls $N=140$ & Patients N = 207 & p-value \\
\hline Age, median (interquartile) & $57(51-63)$ & $51(45-59)$ & $<0.001$ \\
\hline $\mathrm{BMI} \geq 25$ & $92(66 \%)$ & $163(79 \%)$ & 0.02 \\
\hline Male, $\mathrm{N}(\%)$ & $64(46)$ & $129(62)$ & 0.002 \\
\hline Chronic pancreatitis, N (\%) & 0 & $114(55)$ & NA \\
\hline Diabetes, N (\%) & $18(13)$ & $40(19)$ & $0.1^{*}$ \\
\hline Tobacco, N (\%) & $110(79)$ & $136(66)$ & $0.020^{\star}$ \\
\hline Alcohol, N (\%) & $84(60)$ & $120(58)$ & $0.7^{*}$ \\
\hline Gall stone, $\mathrm{N}(\%)$ & 0 & $13(6)$ & NA \\
\hline Tumor, N (\%) & 0 & $18(9)$ & NA \\
\hline
\end{tabular}

TABLE 1: Distribution of age, gender, body mass index (BMI), etc. in case vs. control group with p-values.

In pancreatic cyst patients, $85 \%$ of older patients ( $\geqslant 65$ years old) and $70 \%$ of patients $<65$ years had BMI $\geqslant 25$ $(\mathrm{p}=0.06)$. There was no significant difference between patients with pseudocysts and pancreatic cystic neoplasms with regard to demographic or clinical history.

The patients with chronic pancreatitis were significantly younger than patients without chronic pancreatitis ( 49 vs. 54 years old), tended to be men (70\% vs. $53 \%$ ), were less likely to have diabetes mellitus (11\% vs. $29 \%$ ), and more likely to have gallstones (10\% vs. $2 \%$ ) (Table 2 ). 


\section{Cureus}

\begin{tabular}{|c|c|c|c|}
\hline & No pancreatitis $\mathrm{N}=93$ & Pancreatitis $\mathrm{N}=114$ & p-value \\
\hline Age, median (interquartile) & $54(47-65)$ & $49(44-56)$ & 0.002 \\
\hline Body Mass Index (BMI) & 68 (73\%) & $83(72 \%)$ & 0.9 \\
\hline Male, N (\%) & 49 (53\%) & $80(70 \%)$ & 0.01 \\
\hline Diabetes, N (\%) & 27 (29\%) & $13(11 \%)$ & 0.001 \\
\hline Tobacco, N (\%) & $30(30 \%)$ & $41(36 \%)$ & 0.6 \\
\hline Alcohol, N (\%) & $34(37 \%)$ & $53(46 \%)$ & 0.2 \\
\hline Gall stone, N (\%) & $2(2 \%)$ & $11(10 \%)$ & 0.03 \\
\hline
\end{tabular}

TABLE 2: Distribution of demographic and clinical variables by history of chronic pancreatitis.

Finally, the patients were evaluated in five groups according to the year of diagnosis in the five-year interval. For the first group (those diagnosed before 1997), we had no overweight patients. The incidence of being overweight among the case group was $83 \%$ during the last five-year interval (2008 to 2012, $p=0.009$ ). The rate of being overweight (BMI $\geqslant 25$ ) from 1993 to 2012 has increased from $72 \%$ to $83 \%$, respectively. The frequency rate of pancreatic cysts among our patients has increased from $1.4 \%$ to $38.1 \%$ (Figure 1).

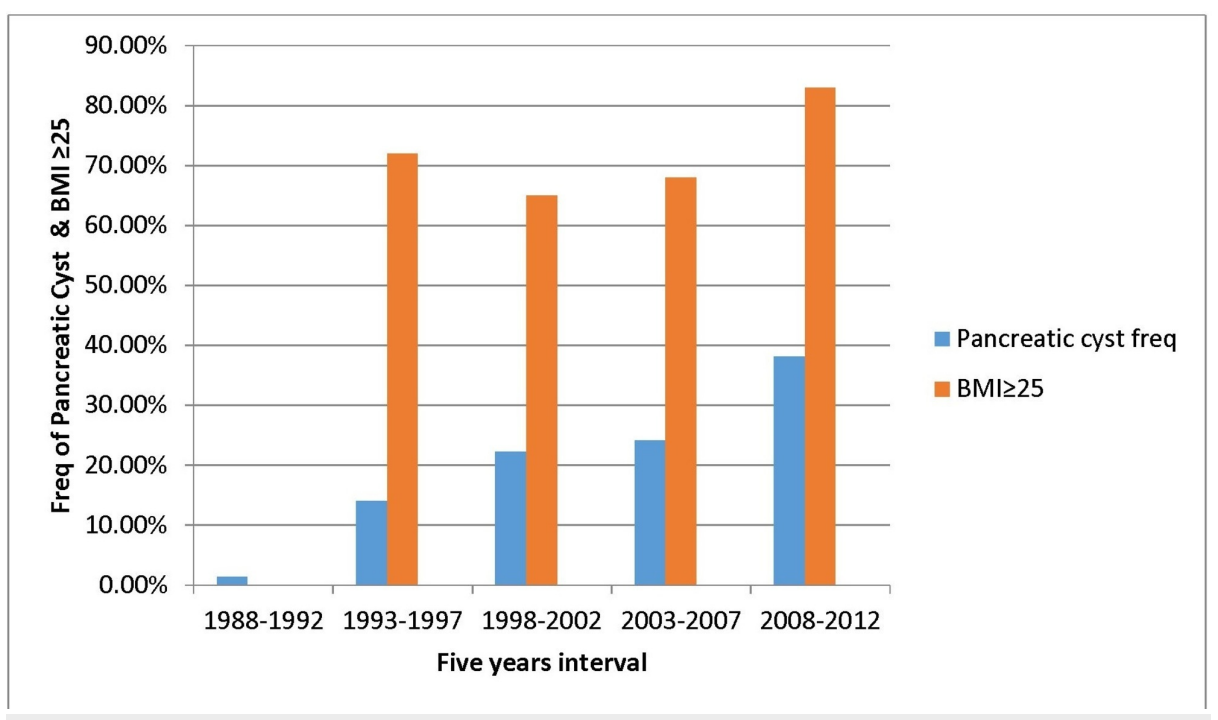

FIGURE 1: Pancreatic cyst frequency and body mass index (BMI) according to the year of diagnosis in five years interval.

\section{Discussion}

This study evaluated the incidence of obesity-overweight among African American patients with pancreatic cysts and demonstrated that the incidence of obesity has increased among patients with pancreatic cysts from 2008 to 2012. Our study showed a significant BMI difference between the two groups. However, the incidence of being overweight (BMI $\geqslant 25$ ) was not the same in different five-year intervals. During the interval from 1993 to 2012, the incidence of overweight status increased. Pancreatic cyst patients had the higher incidence of being overweight from 2008 to 2012.

We had some limitations for selecting the control group in this study. The rate of obesity in the general population of African Americans published by the Centers for Disease Control and Prevention increased from 54\% in 1994 to $69 \%$ in 2012 [10]. It demonstrates the rate of obesity among patients with pancreatic cysts is higher than that of the general population and assumes obesity is a risk factor for the development of pancreatic cysts even without a history of chronic pancreatitis. However, to prove this hypothesis, we require an additional study with a large number of patients in the test and control groups gathered from more recent years. In elderly patients (i.e., those aged $\geqslant 65$ years), obesity's effect as a risk factor to develop pancreatic cysts is greater than in other patient age groups. In our pancreatic cyst patients, $85 \%$ of patients $\geqslant$ 
65 years had $\mathrm{BMI} \geqslant 25$, which was more than the incidence of obesity in the general African American population.

Although pseudocysts and cystic tumors have different pathophysiology, obesity can play a big role in cyst production and development. Pancreatic cancers are more likely to exist in men than in women and more in African Americans than in whites [11,12]. Smoking increases one's risk of pancreatic cancer two to three folds. Alcohol consumption is also considered a risk factor for pancreatic cancer [13-19]. In acute and chronic pancreatitis, obesity is a significant prognostic factor during hospitalization, and BMI independently predicts complications $[20,21]$.

Visceral adipose tissue (VAT) volume is strongly correlated with the formation of a pseudocyst and with systemic inflammatory response syndrome in patients with acute-chronic pancreatitis. Indeed, high VAT volume may lead to severe acute-chronic pancreatitis [7,22].

Obesity in the United States has been increasingly cited as a major health issue in recent decades [23], and our study shows that the rate of pancreatic cysts and its association with obesity is also increasing in recent decades. Currently, the detection rate of pancreatic cysts has increased due to the utilization of imaging facilities. However, the rate of obesity has increased in the United States, too. Therefore, it is difficult to disregard the role of obesity in pancreatic cyst formation because of the concurrent increase in the prevalence of obesity.

\section{Conclusions}

Given the increasing proportion of obese pancreatic cyst patients in recent decades compared to the proportion noted in the 1990s, obesity plays a large role in the formation of pancreatic cysts.

\section{Additional Information}

\section{Disclosures}

Human subjects: All authors have confirmed that this study did not involve human participants or tissue. Animal subjects: All authors have confirmed that this study did not involve animal subjects or tissue. Conflicts of interest: In compliance with the ICMJE uniform disclosure form, all authors declare the following: Payment/services info: Disclosure : This project funded and supported by NIH The authors declare no conflict of interest . Financial relationships: All authors have declared that they have no financial relationships at present or within the previous three years with any organizations that might have an interest in the submitted work. Other relationships: All authors have declared that there are no other relationships or activities that could appear to have influenced the submitted work.

\section{References}

1. Spinelli KS, Fromwiller TE, Daniel RA, et al.: Cystic pancreatic neoplasms: observe or operate. Ann Surg. 2004, 239:651-659. 10.1097/01.sla.0000124299.57430.ce

2. Nugent FW, Stuart K: Adjuvant and neoadjuvant therapy in curable pancreatic cancer . Surg Clin North Am. 2010, 90:323-339. 10.1016/j.suc.2009.12.010

3. Fasanella KE, McGrath K: Cystic lesions and intraductal neoplasms of the pancreas. Best Pract Res Clin Gastroenterol. 2009, 23:35-48. 10.1016/j.bpg.2008.11.011

4. Castillo FC, Targarona J, Thayer SP, Rattner D, Brugge W, Warshaw A: Incidental pancreatic cysts: clinicopathologic characteristics and comparison with symptomatic patients. Arch Surg. 2003, 138:427-434. 10.1001/archsurg.138.4.427

5. Hammel PR, Vilgrain V, Terris B, et al.: Pancreatic involvement in Von Hippel-Lindau disease . Gastroenterology. 2000, 119:1087-1095. 10.1053/gast.2000.18143

6. Gukovsky I, Li N, Todoric J, Gukovskayaet A, Karin M: Inflammation, autophagy, and obesity: common features in the pathogenesis of pancreatitis and pancreatic cancer. Gastroenterology. 2013, 144:1199-1209. 10.1053/j.gastro.2013.02.007

7. Yashima $\mathrm{Y}$, Isayama $\mathrm{H}$, Tsujino $\mathrm{T}$, et al.: A large volume of visceral adipose tissue leads to severe acute pancreatitis. J Gastroenterol. 2011, 46:1213-1218. 10.1007/s00535-011-0430-x

8. Hutley L, Prins JB: Fat as an endocrine organ: relationship to the metabolic syndrome. Am J Med Sci. 2005, 330:280-289. 10.1097/00000441-200512000-00005

9. Nishimura S, Manabe I, Nagasaki M, et al.: In vivo imaging in mice reveals local cell dynamics and inflammation in obese adipose tissue. J Clin Invest. 2008, 118:710-721.

10. Ogden C, Carroll M, Kit Brian K, Flegal K: Prevalence of childhood and adult obesity in the United States, 2011-2012. JAMA. 2014, 311:806-814. 10.1001/jama.2014.732

11. Nahon S, Lahmek P, Lesgourgues B, Nahon-Uzan K, Tuszynski T, Traissac L, Delas N: Predictive factors of GI lesions in 241 women with iron deficiency anemia. Am J Gastroenterol. 2002, 97:590-593. 10.1111/j.15720241.2002.05534.x

12. Sinner PJ, Schmitz KH, Anderson KE, Folsom A: Lack of association of physical activity and obesity with incident pancreatic cancer in elderly women. Cancer Epidemiol Biomarkers Prev. 2005, 14:1571-1573. 10.1158/1055-9965.EPI-05-0036

13. Patel AV, Rodriguez C, Bernstein L, Chao A, Thun M, Calle E: Obesity, recreational physical activity, and risk of pancreatic cancer in a large US cohort. Cancer Epidemiol Biomarkers Prev. 2005, 14:459-466. 10.1158/1055-9965.EPI-04-0583 
14. Aune D, Greenwood DC, Chan DSM, et al.: Body mass index, abdominal fatness and pancreatic cancer risk: a systematic review and non-linear dose-response meta-analysis of prospective studies. Ann Oncol. 2012, 23:843-852. 10.1093/annonc/mdr398

15. Nothlings U, Wilkens LR, Murphy SP, Hankin JH, Henderson BE, Kolonel LN: Body mass index and physical activity as risk factors for pancreatic cancer: the multiethnic cohort study. Cancer Causes Control. 2007, 18:165-175. 10.1007/s10552-006-0100-0

16. Luo JH, Iwasaki M, Inoue M, et al.: Body mass index, physical activity and the risk of pancreatic cancer in relation to smoking status and history of diabetes: a large-scale population-based cohort study in Japan The JPHC study. Cancer Causes Control. 2007, 18:603-612. 10.1007/s10552-007-9002-z

17. Stolzenberg-Solomon RZ, Adams K, Leitzmann M, et al.: Adiposity, physical activity, and pancreatic cancer in the National Institutes of Health-AARP diet and health cohort. Am J Epidemiol. 2008, 167:586-597. 10.1093/aje/kwm361

18. Larsson SC, Orsini N, Wolk A: Body mass index and pancreatic cancer risk: a meta-analysis of prospective studies. Int J Cancer. 2007, 120:1993-1998. 10.1002/ijc.22535

19. Michaud DS, Giovannucci E, Willett WC, Colditz G, Stampfer M, Fuchs C: Physical activity, obesity, height, and the risk of pancreatic cancer. JAMA. 2001, 286:921-929. 10.1001/jama.286.8.921

20. Halonen KI, Leppaniemi AK, Puolakkainen PA, Lundin LE, Kemppainen EA, Hietaranta AJ, Haapiainen RK: Severe acute pancreatitis: prognostic factors in 270 consecutive patients . Pancreas. 2000, 21:266-271.

21. Johnson CD, Toh SKC, Campbell MJ: Combination of APACHE-II score and an obesity score (APACHE-O) for the prediction of severe acute pancreatitis. Pancreatology. 2004, 4:1-6. 10.1159/000077021

22. Martinez J, Sánchez-Payá J, Palazón JM, Aparicio J, Picó A, Pérez-Mateo M : Obesity: a prognostic factor of severity in acute pancreatitis. Pancreas. 1999, 19:15-20.

23. Laiyemo AO: The risk of colonic adenomas and colonic cancer in obesity . Best Pract Res Clin Gastroenterol. 2014, 28:655-663. 10.1016/j.bpg.2014.07.007 